\title{
Maternal Hyperoxygenation Testing in Fetuses with Hypoplastic Left-Heart Syndrome: Association with Postnatal Atrial Septal Restriction
}

\author{
Christopher Mardy $^{a} \quad$ Michelle Kaplinski ${ }^{a}$ b $\quad$ Lynn Peng $^{a} \quad$ Yair J. Blumenfeld ${ }^{b, c}$ \\ David M. Kwiatkowski ${ }^{a}$ Theresa A. Tacy ${ }^{a}$ b Shiraz A. Maskatia ${ }^{a}$ b \\ aDivision of Cardiology, Department of Pediatrics, Stanford University School of Medicine, Stanford, CA, USA; \\ ${ }^{\mathrm{b}}$ Fetal and Pregnancy Health Program, Lucile Packard Children's Hospital, Palo Alto, CA, USA; 'Department of \\ Obstetrics and Gynecology, Stanford University School of Medicine, Stanford, CA, USA
}

\section{Mini-Summary}

- What does this study add to current knowledge?

- Neonates with hypoplastic left-heart syndrome and restrictive atrial septum (RAS) continue to have poor outcomes, often requiring emergent atrial septoplasty (EAS) for stabilization.

- Prenatal prediction of RAS is challenging.

- Prior literature suggests these high-risk fetuses may be identified with (a) pulmonary vein (Pvein) prograde:retrograde velocity time integral (VTI) ratio $<3.0$ and/or (b) with maternal hyperoxygenation $(\mathrm{MHO})$, a decrease in fetal pulmonary artery pulsatility index (PA PI) of $<10 \%$ from baseline.

- What are the main clinical implications?

- Prenatal prediction of RAS in these fetuses is essential for appropriate counseling and for preparation for possible emergent postnatal intervention.

- Pvein prograde:retrograde VTI ratio appears to more accurately identify neonates who undergo EAS than the PA PI and is more reliably measured between observers.

- Fetuses with “intermediate" Pvein prograde:retrograde VTI ratios at baseline (between 3 and 7) are likely to benefit most from MHO testing.

\section{Keywords}

Fetal echocardiography · Maternal hyperoxygenation ·

Hypoplastic left-heart syndrome · Restrictive atrial septum · Emergent atrial septoplasty

\section{Abstract \\ Introduction: In fetuses with hypoplastic left-heart syn- drome (HLHS), maternal hyperoxygenation (MHO) may aid risk stratification. We hypothesized that pulmonary vein}

karger@karger.com www.karger.com/fdt

Karger $\stackrel{\text { ' }}{5}$
C 2021 The Author(s).

Published by S. Karger AG, Basel

This is an Open Access article licensed under the Creative Common Attribution-NonCommercial-4.0 International License (CC BY-NC) (http://www.karger.com/Services/OpenAccessLicense), applicable to the online version of the article only. Usage and distribution for commercial purposes requires written permission.
(Pvein) velocity time integral (VTI) change with $\mathrm{MHO}$ would more reliably identify neonates who undergo emergent atrial septoplasty (EAS) than changes in pulmonary arterial pulsatility index (PA PI). Methods: Fetuses with HLHS who underwent $\mathrm{MHO}$ testing at our institution between 2014 and 2019 were identified. Data were reviewed in a blinded, retrospective manner. Pvein VTI ratio (prograde:retrograde) was calculated. The primary outcome was neonatal EAS. $R \boldsymbol{e}$ sults: Twenty-seven HLHS fetuses underwent MHO, and 5 (19\%) underwent EAS. Without MHO, a Pvein VTI ratio $<3$ 
conferred $60 \%$ sensitivity and $100 \%$ specificity for EAS. With $\mathrm{MHO}$, a Pvein VTI ratio $<6.5$ conferred $100 \%$ sensitivity and specificity. For an intermediate group of fetuses with a baseline Pvein VTI ratio 3-7, the ratio decrease with MHO conferred $100 \%$ sensitivity and specificity. Compared to the Pvein VTI ratio, PA PI was less accurate in identifying EAS neonates. Discussion/Conclusion: Addition of MHO appears to improve the diagnostic ability of the Pvein VTI ratio to identify HLHS fetuses who undergo EAS. The Pvein VTI ratio change may more accurately identify fetuses who undergo EAS than change in PA PI and has less interobserver variability.

(c) 2021 The Author(s).

Published by S. Karger AG, Basel

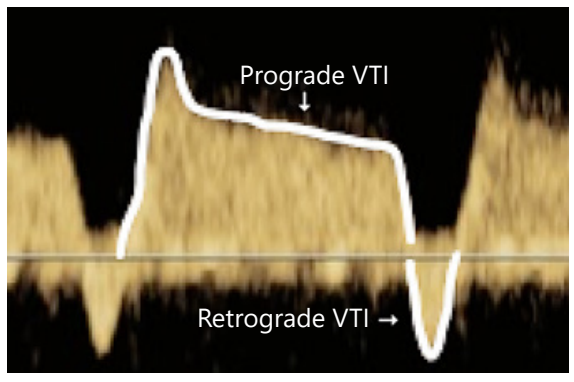

Fig. 1. Pvein Doppler pattern in a fetus with an unrestrictive atrial septum. The calculated Pvein VTI ratio (prograde VTI)/(retrograde VTI) is approximately 10 . Pvein, pulmonary vein; VTI, velocity time integral.

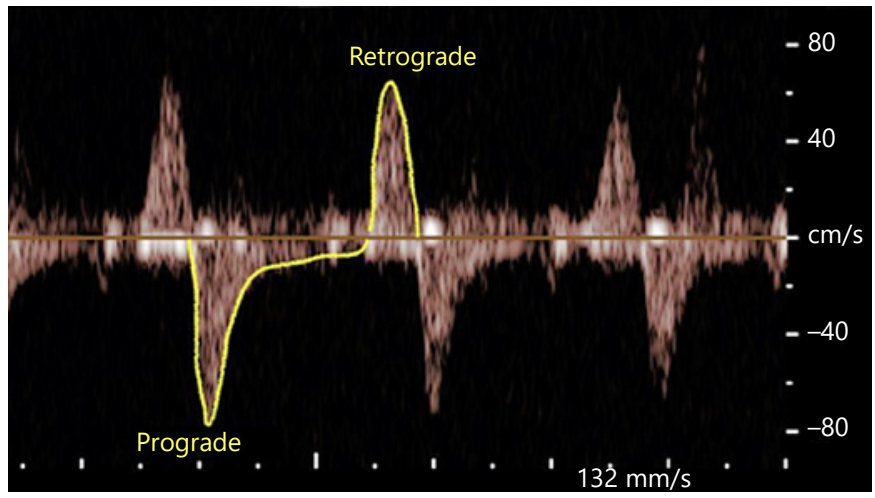

Fig. 2. Pvein Doppler: fetus with atrial septal restriction (Pvein VTI ratio between 1 and 2). Pvein, pulmonary vein; VTI, velocity time integral. and/or lymphatic obstruction may further complicate subsequent care [10]. Prenatal diagnosis of RAS is therefore essential to risk stratification, counseling, and early neonatal management, in addition to the evaluation for potential fetal cardiac intervention [11]. Preand postnatal atrial septal interventions require a vast array of subspecialists, and multidisciplinary preparedness is critical.

Prenatal prediction of RAS can be challenging. Marked abnormalities in pulmonary venous Doppler patterns on fetal echocardiogram (shown in Fig. 1,2) may identify HLHS fetuses with RAS, but more subtle findings may lead fetuses to be underdiagnosed. A high degree of atrial septal restriction may be underappreciated in fetal life due to relatively low flow through the pulmonary vascular bed in utero; pulmonary venous hypertension and development of lymphatic lung disease may clinically manifest only after delivery $[12,13]$.

Maternal hyperoxygenation (MHO) has been proposed to aid in prenatal detection of RAS [14]. MHO increases fetal oxygen content, leads to pulmonary vasodila-

Maternal Hyperoxygenation Testing in Fetal Hypoplastic Left-Heart Syndrome
Fetal Diagn Ther 2021;48:678-689 DOI: $10.1159 / 000519322$ 


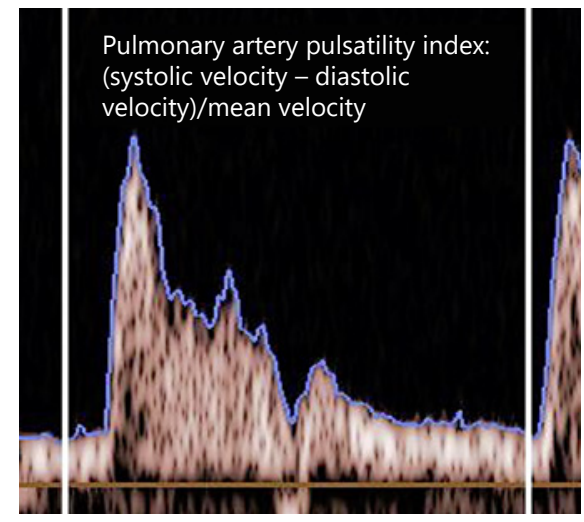

Fig. 3. Pulmonary artery pulsatility index.

accurate retrograde Doppler signal. We hypothesized that change with MHO in Pvein VTI net would best identify fetuses who underwent EAS after birth.

\section{Materials and Methods}

\section{Patient Population}

Approval was obtained from the Stanford University Institutional Review Board. Pregnant women carrying fetuses with HLHS between 2014 and 2019 were retrospectively identified from the Fetal Cardiology Program across Stanford Children's Health sites and were included if they underwent $\mathrm{MHO}$ and delivered at our institution. Our large volume system performs over 3,000 fetal echocardiograms annually, and we have aimed to perform MHO in all HLHS fetuses since 2014. Fetal and postnatal echocardiograms were reviewed, and clinical data were extracted from the medical record. All forms of HLHS were included, as was mitral atresia and ventricular septal defect, due to similar potential concern for RAS. Although our center does not offer fetal intervention for RAS, it is our practice to offer referral to an interventional center when appropriate.

\section{MHO Testing}

Typically, our institution performs echocardiograms with MHO testing at approximately 34 weeks' gestation in HLHS fetuses when neonatal intervention is planned. Baseline measures are performed. Oxygen is then administered to mothers via nonrebreather facemask at $8 \mathrm{~L}$ per minute for $10 \mathrm{~min}$, and select measures are repeated.

\section{Echocardiographic Parameters}

Fetal echocardiograms (Acuson SC2000, Siemens Medical Solutions USA Inc., Mountain View, CA, USA, Philips Epiq 7, or Philips IE33, Philips Medical Systems, Bothell, WA, USA) were retrospectively measured by a single observer blinded to outcome (CM) after specific training by a fetal echocardiography expert (S.A.M.). Interobserver reliability testing was performed on $30 \%$ of measurements.

Percent response was calculated as percent change from baseline to MHO states where appropriate. Atrial septal gradient was recorded at baseline and with MHO. Overall assessment of RAS by the fetal cardiologist, including qualitative and quantitative measures from prior literature, was noted. A PA spectral Doppler pattern was obtained at the artery's midpoint. The pulsatility index was calculated:

$$
\text { Pulsatility index }=\left(\frac{\text { systolic velocity }- \text { diastolic velocity }}{\text { mean velocity }}\right) .
$$

Pvein VTI was measured over one cardiac cycle during a period of consistent Doppler pattern at baseline and after MHO. For both PA and Pvein Doppler patterns, we chose the best quality pattern and angle of insonation for measurement. If angles of insonation were equivalent, both lungs were sampled, and the results were averaged. The same side was sampled at baseline and with $\mathrm{MHO}$ within each patient. Prograde and retrograde Pvein VTI were recorded, and their ratio and net difference were calculated as below. "Prograde" and "retrograde" have been referred to "forward" and "reverse," respectively, in the literature.

$$
\begin{aligned}
& \text { Pulmonary vein VTI ratio }=\left(\frac{\text { Pvein VTI prograde }}{\text { Pvein VTI retrograde }}\right) . \\
& \text { Pulmonary vein VTI Net }=\text { Pvein VTI prograde - } \\
& \text { Pvein VTI retrograde }
\end{aligned}
$$

The primary outcome was postnatal EAS. Atrial septoplasty was considered emergent if a catheter-based or surgical intervention was performed for the purpose of relieving atrial septal restriction. Early surgical intervention to address other physiologies (e.g., pulmonary overcirculation) was not considered EAS for the purposes of this study.

Laboratory data were reviewed, noting the lowest, highest, and median $\mathrm{P}_{\mathrm{a}} \mathrm{O}_{2}$ prior to any intervention and lowest arterial $\mathrm{pH}$, lowest bicarbonate level, and highest lactate (discarding the first level). We employed a composite outcome adapted from Gellis et al. [7] which was met with any one of the following: $\mathrm{pH}<7.15$, bicarbonate $<16 \mathrm{mmol} / \mathrm{L}$, lactate $>5 \mathrm{mmol} / \mathrm{L}$, or median $\mathrm{SpO}_{2}<60 \%$ in the first $2 \mathrm{~h}$ of life. The first postnatal echocardiogram was reviewed (CM). Qualitative assessment of RAS was based on the interpretation documented at the time of the study. Atrial septal defect (ASD) gradient was measured across 2 cardiac cycles.

Statistical analyses were performed using SPSS Statistics version 26 (IBM Corporation, Armonk, NY). Kolmogorov-Smirnov and Shapiro-Wilk tests were used to test for normality. Parametric data are presented as mean \pm standard deviation and nonparametric data as median (interquartile range). Paired $t$ tests compared means of normally distributed variables, and MannWhitney $U$ tests compared medians of nonparametric variables. Pearson's and Spearman's correlations were employed for parametric and nonparametric continuous variables, respectively. $\chi^{2}$ tests compared categorical variables, with the Fisher's exact test when observations $<5$. A $p$ value of $<0.05$ was considered statistically significant across tests. Receiver-operating characteristic (ROC) curves were generated, with EAS as state variable. If there was no Pvein VTI retrograde deflection, the subject was removed for statistical comparisons of the Pvein VTI ratio, but for ROC analysis, a value matching the cohort's maximum ratio was used. Youden's index was used to identify optimal cutpoints for the state variable. 


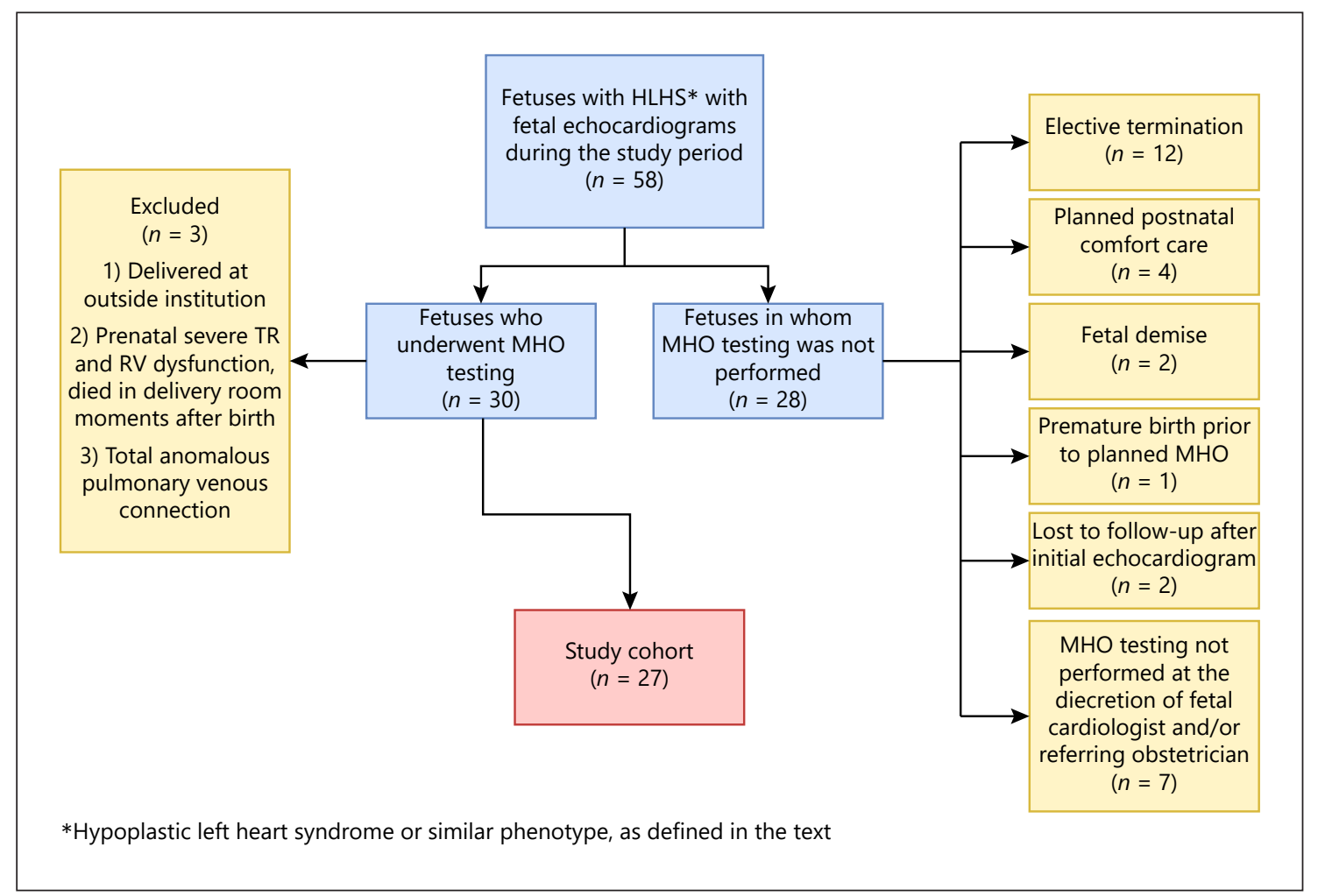

Fig. 4. Flow diagram for cohort selection. HLHS, hypoplastic left-heart syndrome; MHO, maternal hyperoxygenation.

\section{Results}

\section{Patients and Overall Outcomes}

Fifty-eight fetuses with HLHS were identified during the study period, and 30 (53\%) underwent MHO testing, with 3 additional fetuses excluded (Fig. 4). Of the $28 \mathrm{fe}-$ tuses not undergoing $\mathrm{MHO}$ testing, there were 12 elective terminations, 2 fetal demises, and 4 planned for postnatal comfort care. Seven fetuses did not undergo MHO testing at the discretion of the fetal cardiologist, mainly in the first year of the study period. The remaining 27 (47\%) fetuses comprised the study cohort. There were no maternal adverse events with administration of oxygen.

Patient characteristics and overall outcomes are provided in Table 1 . No fetal interventions were performed. Of the 27 fetuses in our cohort, 7 (26\%) were prenatally diagnosed with RAS based on overall assessment by the fetal cardiologist, and of these, 4 underwent postnatal EAS. One additional patient underwent EAS, who was thought prenatally to be low-risk ( 5 total EAS, 19\%). Four of these 5 neonates underwent EAS on the first day of life, with one neonate undergoing EAS on the fifth day of life, having had relative hypoxia with an initially underappreciated degree of RAS. All 5 neonates who underwent EAS died prior to discharge, median 53 days (range 9-166 days). Of the 22 non-EAS neonates, 19 (86\%) survived to discharge. There were major comorbidities for 5 (all 3 of the non-EAS neonates and 2 of the 5 EAS neonates) of the 8 deaths in the entire cohort (Table 2). Postnatally, some degree of RAS (including mild) was noted on echocardiogram in 15 of 27 fetuses (56\%), which included 6 of the 7 prenatal RAS diagnoses. Survival for those with any degree of RAS on the postnatal echocardiogram was 53\% $(8 / 15)$ compared to $92 \%(11 / 12)$ in those without RAS $(p=0.03)$. Survival for patients with mild RAS (i.e., some degree of RAS but not undergoing EAS) was $80 \%(8 / 10)$. No patient had an intact atrial septum. Neither of the 2 patients with mitral atresia and ventricular septal defect underwent EAS. Clinical data for EAS and non-EAS groups are summarized in Table 3.

\section{Predictors of Postnatal RAS}

Compared to non-EAS fetuses, EAS fetuses had a lower Pvein VTI ratio at baseline and with $\mathrm{MHO}$ and lower 
Table 1. Overall descriptive data for entire cohort

\begin{tabular}{|c|c|}
\hline \multicolumn{2}{|l|}{ Prenatal } \\
\hline Sex, $n(\%)$ & Female = $11(41)$ \\
\hline Gestational age at $\mathrm{MHO}$ testing, weeks & $34.15 \pm 1.60$ \\
\hline RV systolic function, $n(\%)$ & $\begin{array}{l}\text { Normal }=26(98) \\
\text { Moderately depressed }=1(4)\end{array}$ \\
\hline Tricuspid regurgitation severity, $n(\%)$ & $\begin{array}{l}\text { None = } 15(56) \\
\text { Trivial = } 5(19) \\
\text { Mild = } 5(19) \\
\text { Moderate = } 2(7)\end{array}$ \\
\hline Prenatal assessment of atrial septum restriction, $n(\%)$ & $\begin{array}{l}\text { Unrestrictive }=20(74) \\
\text { Restrictive }=7(26)\end{array}$ \\
\hline Noncardiac anomalies, $n(\%)$ & $\begin{array}{l}\text { Absent }=20(74) \\
\text { Present }=7(26)\end{array}$ \\
\hline Known genetic diagnosis, $n(\%)$ & $\begin{array}{l}\text { Absent }=25(93) \\
\text { Present }=2(7)\end{array}$ \\
\hline HLHS phenotype, $n$ (\%) & $\begin{array}{l}\text { MS/AS }=6(22) \\
M S / A A=9(33) \\
M A / A A=10(37) \\
\text { MA/VSD/AS 2 (7) }\end{array}$ \\
\hline \multicolumn{2}{|l|}{ Postnatal } \\
\hline Birth weight, kg & $3.08 \pm 0.44$ \\
\hline Postnatal assessment of atrial septum restriction, $n(\%)$ & $\begin{array}{l}\text { Unrestrictive = } 12(44) \\
\text { Restrictive = } 15(56)\end{array}$ \\
\hline $\mathrm{EAS}, n(\%)$ & $\begin{array}{l}\text { No EAS = } 22(82) \\
E A S=5(19)\end{array}$ \\
\hline Survival to discharge, $n(\%)$ & $\begin{array}{l}\text { Survived = } 19(70) \\
\text { Died = } 8(30)\end{array}$ \\
\hline
\end{tabular}

$\mathrm{MHO}$, maternal hyperoxia; RV, right ventricle; MS/AS, mitral stenosis/aortic stenosis; MS/ $\mathrm{AA}$, mitral stenosis/aortic atresia; MA/AA, mitral atresia/aortic atresia; MA/VSD/AS, mitral atresia/ventricular septal defect/aortic stenosis; EAS, emergent atrial septoplasty; HLHS, hypoplastic left-heart syndrome.
Pvein VTI net with MHO. In response to MHO, the Pvein VTI ratio increased in the non-EAS group, while the ratio was not significantly different in the EAS group (Table 4 ). No significant changes with MHO were seen in net Pvein VTI, ASD gradient, or PA PI in EAS or non-EAS fetuses. Of the 5 neonates who underwent EAS, 4 had Pvein VTI ratio decrease with $\mathrm{MHO}$ and one increased with MHO.

None of the fetal echocardiographic measures described were associated with postnatal median $\mathrm{O}_{2}$ saturation or $\mathrm{PaO}_{2}$. The Pvein VTI ratio at baseline was inversely associated with the postnatal lactate level $(r=-0.421, p$ $=0.03$ ). None of the baseline measures correlated with postnatal ASD gradient, but the Pvein VTI ratio during MHO $(r=-0.474, p=0.02)$ and percent change in the
Pvein VTI ratio with MHO $(r=-0.497, p=0.01)$ was inversely correlated with the postnatal ASD gradient. Fetuses who met the composite outcome were more likely to undergo EAS (Table 3) and had a lower Pvein VTI ratio at baseline $(6.59+/-3.51$ vs. $11.08+/-4.16, p=0.01)$ and with $\mathrm{MHO}(7.48+/-3.84$ vs. $14.36+/-7.90, p=0.02)$.

Table 5 summarizes the performance for the tested prenatal variables in identifying fetuses who underwent postnatal EAS. Generally, Pvein VTI ratio parameters outperformed net Pvein VTI response and PA PI response. Optimal accuracy (100\% sensitivity and 100\% specificity) was achieved using a Pvein VTI ratio with $\mathrm{MHO}<6.5$. The following tiered approach also conferred $100 \%$ sensitivity and specificity for EAS: ascribing fetus- 
Table 2. Description of clinical events for patients who died prior to discharge

\begin{tabular}{|c|c|c|c|c|c|}
\hline $\begin{array}{l}\text { Patient } \\
\text { No. }\end{array}$ & $\begin{array}{l}\text { HLHS } \\
\text { anatomy }\end{array}$ & $\begin{array}{l}\text { Age at } \\
\text { intervention, } \\
\text { days }\end{array}$ & $\begin{array}{l}\text { Type of } \\
\text { emergent atrial } \\
\text { intervention }\end{array}$ & $\begin{array}{l}\text { Age at } \\
\text { death, } \\
\text { days }\end{array}$ & Notes \\
\hline 2 & MS/AA & 0 & Atrial stent & 35 & $\begin{array}{l}\text { After EAS, underwent hybrid stage } 1 \text { on the first day of life. Multiple } \\
\text { episodes of coronary insufficiency, concerning for LV-dependent } \\
\text { coronary circulation, prompting transplant and VAD evaluation. Died } \\
\text { due to sudden bradycardic arrest }\end{array}$ \\
\hline 3 & MS/AA & 0 & Atrial stent & 53 & $\begin{array}{l}\text { An atrial stent complicated by atrial perforation, cannulated to } \\
\text { VA-ECMO, with atrial repair, and septectomy with PA banding. } \\
\text { Unable to wean from ECMO, converted to VAD support. Persistent } \\
\text { vasodilatory shock and end organ dysfunction. Care withdrawn }\end{array}$ \\
\hline 4 & MS/AA & 0 & Atrial stent & 89 & $\begin{array}{l}\text { Norwood at } 6 \text { days, arrest on the first postoperative day. Multiorgan } \\
\text { system dysfunction, chronic diastolic heart failure, and systolic } \\
\text { dysfunction. Developed a large ischemic stroke prompting } \\
\text { withdrawal of care }\end{array}$ \\
\hline 5 & MS/AS & 5 & Atrial stent & 9 & $\begin{array}{l}\text { Initially stable after birth but progressive acidosis and hypoxia } \\
\text { prompting emergent stenting. VACTERL with multicystic dysplastic } \\
\text { kidneys with renal failure; weighing long term prognosis, the medical } \\
\text { team and family opted not to pursue surgical palliation }\end{array}$ \\
\hline 6 & MS/AS & $\mathrm{n} / \mathrm{a}$ & $\mathrm{n} / \mathrm{a}$ & 5 & $\begin{array}{l}\text { Recalcitrant fetal SVT prompted delivery. Postnatal diagnosis of PJRT. } \\
\text { Unable to be managed medically due to severe renal dysfunction. } \\
\text { Care withdrawn }\end{array}$ \\
\hline
\end{tabular}

MS/AS, mitral stenosis/aortic stenosis; MS/AA, mitral stenosis/aortic atresia; MA/AA, mitral atresia/aortic atresia; PVR, pulmonary vascular resistance; VA-ECMO, venoatrial extracorporeal membrane oxygenation; VAD, ventricular assist device; SVT, supraventricular tachycardia; PJRT, permanent junctional-reciprocating tachycardia; EAS, emergent atrial septoplasty; HLHS, hypoplastic left-heart syndrome.

es with a baseline Pvein VTI ratio $<3$ to the EAS group, those with a baseline Pvein VTI ratio $\geq 7$ to the non-EAS group and ascribing the remaining fetuses with a Pvein VTI ratio that decreases from baseline to MHO to the EAS group. Interobserver testing was performed on $30 \%$ of measurements, with significantly less interobserver variability in the Pvein VTI ratio (intraclass correlation $>0.9$ ) than in PA PI (intraclass correlation 0.05) (Table 6).

Maternal Hyperoxygenation Testing in Fetal Hypoplastic Left-Heart Syndrome

\section{Discussion/Conclusion}

We performed a single institution, retrospective cohort study of 27 fetuses prenatally diagnosed with HLHS or similar phenotype who underwent MHO testing, to identify fetal parameters associated with postnatal EAS. To our knowledge, our study is the first to compare various methods to assess fetal pulmonary arterial vasoreactivity in response to MHO. In our cohort, the Pvein VTI ratio, both at baseline and with $\mathrm{MHO}$, reliably discrimi- 
Table 3. Postnatal laboratory parameters for intervention and nonintervention groups

\begin{tabular}{lllr}
\hline Postnatal parameter & EAS $(n=5)$ & No EAS $(n=22)$ & $p$ value \\
\hline pH - lowest & $7.14(7.06,7.35)$ & $7.29(7.24,7.33)$ & 0.23 \\
Bicarbonate - lowest, mmol/L & $18(13,19)$ & $19(16,21)$ & 0.21 \\
Lactate - highest, $\mathrm{mmol} / \mathrm{L}$ & $3.6(2.9,6.5)$ & $2.4(2.2,3.3)$ & $\mathbf{0 . 0 3}$ \\
$\mathrm{SpO}_{2}$ - median (first 2 h), \% & $80(53,86)$ & $94(89,95)$ & $<\mathbf{0 . 0 1}$ \\
$\mathrm{P}_{\mathrm{a}}$ - median, $\mathrm{mm} \mathrm{Hg}$ & $40(29,42)$ & $49(46,51)$ & $\mathbf{0 . 0 2}$ \\
ASD gradient, mm Hg & $10.1(5.6,14.8)$ & $3.4(1.0,4.4)$ & $<\mathbf{0 . 0 1}$ \\
Positive composite outcome, $n(\%)^{*}$ & $4(80)$ & $5(23)$ & $\mathbf{0 . 0 3}$ \\
\hline
\end{tabular}

Values expressed as median (IQR). $\mathrm{SpO}_{2}$, saturation measured by pulse oximetry; $\mathrm{P}_{\mathrm{a}} \mathrm{O}_{2}$, partial pressure of oxygen; ASD, atrial septal defect; EAS, emergent atrial septoplasty. Laboratory data included values prior to any intervention (unless otherwise noted). * Composite outcome: "positive" with any of the following: $\mathrm{pH}<7.15$, bicarbonate $<16 \mathrm{mmol} / \mathrm{L}$, lactate $>5 \mathrm{mmol} / \mathrm{L}$, or median $\mathrm{SpO}_{2}<60 \%$ in the first $2 \mathrm{~h}$ of life (adapted from Gellis et al. [7]).

Table 4. Comparing fetal echocardiographic parameters for intervention and nonintervention groups

\begin{tabular}{|c|c|c|c|}
\hline & $\operatorname{EAS}(n=5)$ & No EAS $(n=22)$ & $p$ value \\
\hline \multicolumn{4}{|l|}{ Baseline } \\
\hline Pvein VTI ratio & $3.92 \pm 1.69$ & $10.87 \pm 3.82$ & $<0.01$ \\
\hline Pvein VTI net, m & $0.063 \pm 0.023$ & $0.073 \pm 0.019$ & 0.33 \\
\hline ASD gradient, $\mathrm{mm} \mathrm{Hg}$ & $1.17(0.33,2.01)$ & $0.46(0.22,0.71)$ & 0.45 \\
\hline PA PI & $2.70 \pm 0.80$ & $2.50 \pm 0.59$ & 0.57 \\
\hline \multicolumn{4}{|l|}{$\mathrm{MHO}$} \\
\hline Pvein VTI ratio & $3.68 \pm 1.62$ & $13.91 \pm 6.98$ & $<0.01$ \\
\hline$p$ value (baseline vs. $\mathrm{MHO}$ ) & 0.34 & 0.03 & \\
\hline Pvein VTI net, m & $0.067 \pm 0.026$ & $0.096 \pm 0.028$ & 0.04 \\
\hline$p$ value (baseline vs. $\mathrm{MHO}$ ) & 0.65 & $<0.01$ & \\
\hline ASD gradient, $\mathrm{mm} \mathrm{Hg}$ & $0.65(0.45,2.34)$ & $0.61(0.25,0.97)$ & 0.36 \\
\hline$p$ value (baseline vs. MHO) & 0.35 & 0.89 & \\
\hline PA PI & $2.26 \pm 0.86$ & $2.37 \pm 0.56$ & 0.74 \\
\hline$p$ value (baseline vs. $\mathrm{MHO}$ ) & 0.61 & 0.38 & \\
\hline \multicolumn{4}{|l|}{ Response } \\
\hline Pvein VTI ratio response & $-5.1 \pm 16.5$ & $32.0 \pm 49.7$ & 0.01 \\
\hline Pvein VTI net response & $9.3 \pm 33.1$ & $40.9 \pm 54.9$ & 0.23 \\
\hline ASD gradient response & $44.4(23.1,110.1)$ & $-8.8(-60.8,43.2)$ & 0.52 \\
\hline PA PI response & $0.2 \pm 78.4$ & $1.3 \pm 32.5$ & 0.98 \\
\hline
\end{tabular}

EAS, emergent atrial septoplasty; $\mathrm{MHO}$, maternal hyperoxygenation; Pvein VTI, pulmonary vein velocity time integral; Pvein VTI ratio, (Pvein VTI prograde)/(Pvein VTI retrograde); Pvein VTI net, Pvein VTI prograde - Pvein VTI retrograde; ASD, atrial septal defect; $\mathrm{PA} \mathrm{PI}$, pulmonary artery pulsatility index; Response, percentage change in listed value from baseline to $\mathrm{MHO}$.

nated between patients who received and did not receive EAS. PA PI response to MHO was similar between fetuses in both groups. Fetuses with high-baseline Pvein VTI ratios were unlikely to undergo EAS, those with low baseline ratios were more likely to undergo EAS and for those with intermediate baseline ratios, a decrease in the Pvein VTI ratio with $\mathrm{MHO}$ increased risk of EAS. The current published experience of using $\mathrm{MHO}$ in this context is quite limited. Until larger, multicenter studies can be performed, change in the Pvein VTI ratio should be 
Table 5. Comparison of multiple prenatal predictive variables in predicting EAS

\begin{tabular}{lllll}
\hline & Sensitivity, $\%$ & Specificity, $\%$ & PPV, $\%$ & NPV, \% \\
\hline $\begin{array}{l}\text { Pvein } V T I \text { ratio test } \\
\text { Baseline ratio }<3\end{array}$ & & & & \\
Baseline ratio $<7$ & $60(15,95)$ & $100(85,100)$ & 100 & $92(79,97)$ \\
MHO ratio $<6.5$ & $100(48,100)$ & $82(60,95)$ & $56(34,75)$ & 100 \\
Ratio $\downarrow$ with MHO & $100(48,100)$ & $100(85,100)$ & 100 & 100 \\
Baseline $<3 \rightarrow$ EAS, Baseline $\geq 7 \rightarrow$ non-EAS, & $80(28,99)$ & $71(48,89)$ & $40(23,60)$ & $94(72,99)$ \\
$\quad$ Baseline $>3$ and $<7$ and ratio $\downarrow$ with MHO $\rightarrow$ EAS & $100(48,100)$ & $100(85,100)$ & 100 & 100 \\
\hline $\begin{array}{l}\text { Other variable tests } \\
\text { Net Pvein VTI inc }<35 \%\end{array}$ & $80(29,99)$ & $45(24,68)$ & $25(16,37)$ & $91(62,98)$ \\
PA PI $<10 \%$ response & $25(1,81)$ & $48(26,70)$ & $8(2,34)$ & $77(62,87)$ \\
PA PI $<20 \%$ response & $25(1,81)$ & $24(8,47)$ & $6(1,26)$ & $63(39,81)$ \\
\hline
\end{tabular}

Values expressed as \% $(95 \% \mathrm{Cl})$. Pvein VTI, pulmonary vein velocity time integral; Pvein VTI ratio, (Pvein VTI prograde)/(Pvein VTI retrograde); ratio $\downarrow$ with MHO, Pvein VTI ratio decreases from baseline to MHO state; Net Pvein VTI inc, less than a 35\% increase in net Pvein VTI from baseline to MHO states; PA PI response, percent decrease from baseline to MHO state; PPV, positive predictive value; NPV, negative predictive value; EAS, emergent atrial septoplasty.

Table 6. Interobserver variability for fetal echocardiographic parameters

\begin{tabular}{|c|c|c|c|c|}
\hline & ICC & $95 \% \mathrm{Cl}$ & Mean bias & $p$ value \\
\hline \multicolumn{5}{|l|}{ Pvein VTI ratio } \\
\hline Baseline & 0.913 & $(0.458,0.982)$ & 1.68 & $<0.001$ \\
\hline $\mathrm{MHO}$ & 0.978 & $(0.894,0.996)$ & 1.12 & $<0.001$ \\
\hline \multicolumn{5}{|l|}{ Pvein VTI net } \\
\hline Baseline, m & 0.823 & $(0.189,0.960)$ & 0.002 & 0.015 \\
\hline $\mathrm{MHO}, \mathrm{m}$ & 0.972 & $(0.884,0.994)$ & 0.003 & $<0.001$ \\
\hline \multicolumn{5}{|l|}{$P A P I$} \\
\hline Baseline & 0.047 & $(-3.226,0.785)$ & 0.551 & 0.474 \\
\hline $\mathrm{MHO}$ & 0.84 & $(0.239,0.964)$ & 0.006 & 0.012 \\
\hline \multicolumn{5}{|c|}{$\begin{array}{l}\text { Interobserver testing performed on } 30 \% \text { of cohort's } \\
\text { measurements. ICC, intraclass correlation; Cl, confidence interval; } \\
\text { MHO, maternal hyperoxygenation; Pvein VTI, pulmonary vein } \\
\text { velocity time integral; Pvein VTI ratio, (Pvein VTI prograde)/(Pvein } \\
\text { VTI retrograde); Pvein VTI net, Pvein VTI prograde - Pvein VTI } \\
\text { retrograde; PA PI, pulmonary artery pulsatility index. }\end{array}$} \\
\hline
\end{tabular}

used in conjunction with change in PA PI to assess $\mathrm{MHO}$ response in fetuses with HLHS. Our findings will be helpful for the fetal cardiologist attempting to identify the high-risk subgroup of HLHS fetuses with RAS and may help guide potential referrals for fetal atrial septoplasty, organize coordinated delivery planning, and better prepare the multidisciplinary team receiving this high-risk group of patients.

Maternal Hyperoxygenation Testing in Fetal Hypoplastic Left-Heart Syndrome

\section{Pulmonary Venous Doppler}

While the use of Pvein Doppler to predict EAS has been previously described $[1,15,16]$, the addition of $\mathrm{MHO}$ to this calculus has not been investigated. Increased A wave amplitude can signal relative LA hypertension in the fetus with HLHS and RAS (Fig. 1, 2). We hypothesized that by augmenting pulmonary blood flow and venous return to the LA with $\mathrm{MHO}$, those with significant RAS would have higher LA pressure and reduced augmentation of pulmonary venous return relative to those without RAS. This phenomenon would translate into worsened the Pvein VTI ratio (reflecting increased LA pressure) and lower increase in Pvein VTI net (reflecting reduced augmentation of pulmonary venous return).

The Pvein VTI ratio at baseline and with $\mathrm{MHO}$ was closely associated with EAS. On average, the Pvein VTI ratio increased from baseline to MHO in the non-EAS group, while there was no significant change for the EAS group. The change in the Pvein VTI ratio from baseline to MHO was significantly different between EAS and non-EAS groups (decrease by $5 \%$ vs. increase by $32 \%$, respectively, $p=0.01$ ) (Table 4; Fig. 5). The addition of MHO improved specificity of the Pvein VTI ratio $(<7$ at baseline conferred $82 \%$ specificity, while $<6.5$ with $\mathrm{MHO}$ conferred $100 \%$ specificity), and with ROC analysis, we found the area under the curve (AUC) for the ratio both at baseline and with MHO to be excellent (AUC 0.98 and 1.00, respectively) (Fig. 6). This suggests that while the Pvein VTI ratio performs well at baseline, there may be 


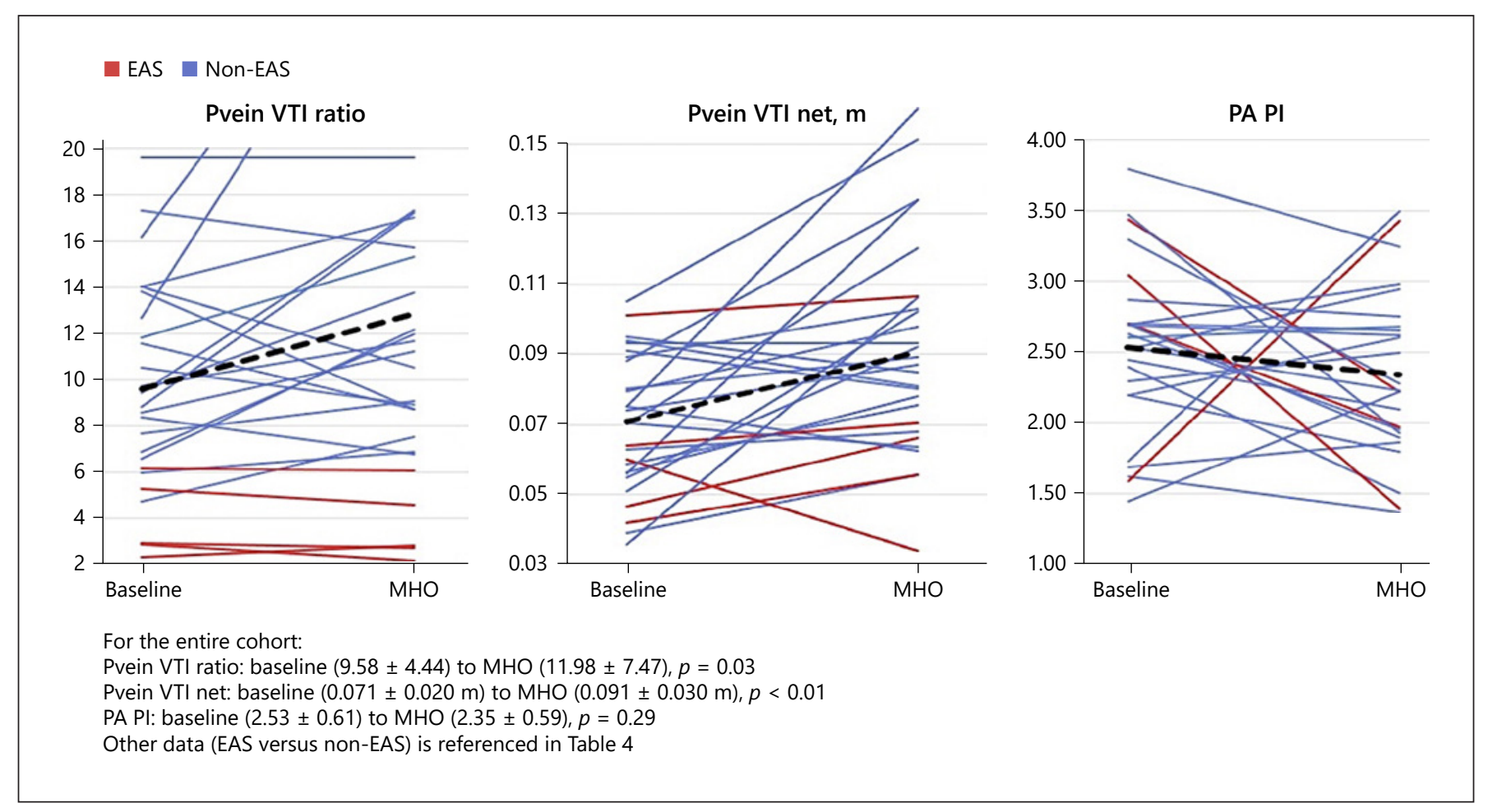

Fig. 5. Line graphs: individual fetus responses from baseline to MHO states in 3 variables. MHO, maternal hyperoxygenation; PA PI, pulmonary artery pulsatility index.

Fig. 6. EAS (ROC) curve. ROC, receiveroperating characteristic; EAS, emergent atrial septoplasty; PA PI, pulmonary artery pulsatility index; MHO, maternal hyperoxygenation; VTI, velocity time integral.

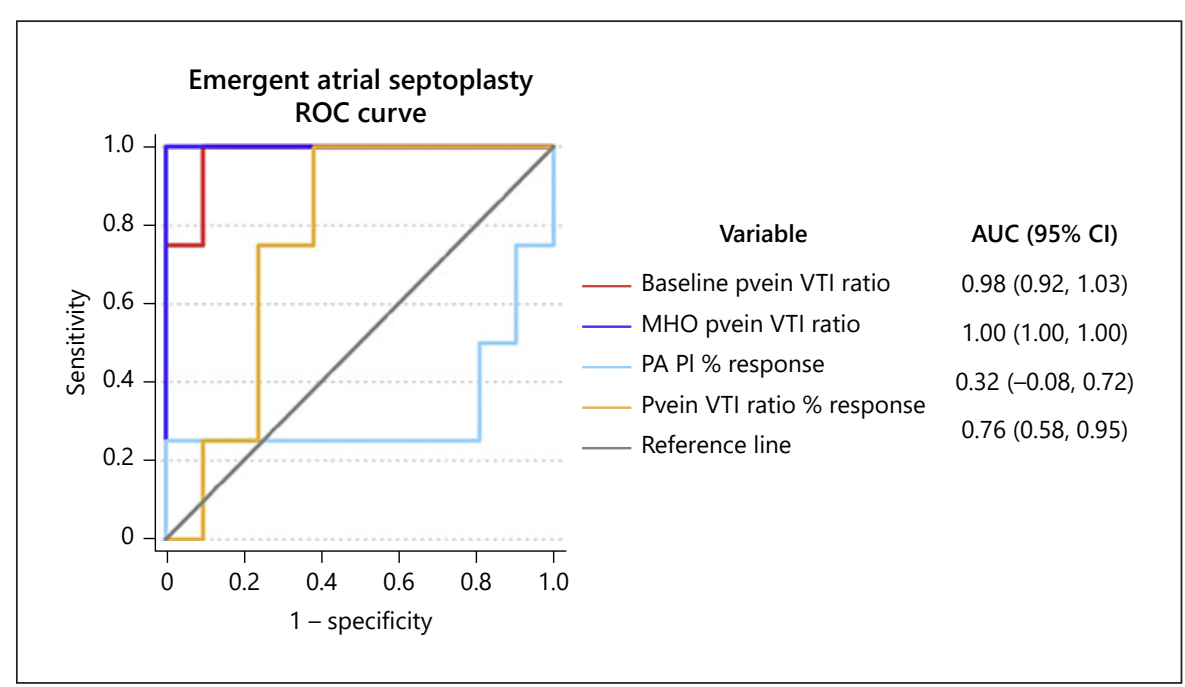

incremental benefit with $\mathrm{MHO}$ to improve specificity. It should be noted that there were 2 non-EAS patients with the Pvein VTI ratio with MHO between 6.5 and 7: one ratio increased and the other decreased with MHO.

In our cohort, the Pvein VTI ratio $<3$ (cutoff from Divanović et al. [16]) conferred 60\% sensitivity, missing 2 fetuses that underwent EAS (whose ratios decreased with $\mathrm{MHO}$ ). Our data suggest the following approach: fetuses that clearly have low baseline Pvein VTI ratios $(<3)$ should be considered high-risk for requiring EAS, regardless of response to MHO. Those with high Pvein VTI ratios $(\geq 7)$ are very unlikely to require EAS. There is likely no substantial benefit to $\mathrm{MHO}$ testing in these groups. However, the "intermediate group" of fetuses 
(baseline Pvein VTI ratio of 3:7) may benefit the most from MHO testing as Pvein VTI ratio response with MHO may offer improved accuracy in identifying neonates who undergo EAS.

Although there was a difference in Pvein VTI net between non-EAS and EAS groups with the addition of $\mathrm{MHO}$, the Pvein VTI ratio at baseline and with MHO differentiated EAS and non-EAS groups with greater accuracy than Pvein VTI net. This may be because differences in retrograde VTI result in larger differences in Pvein VTI ratio compared to Pvein VTI net. Additionally, minor differences in the insonation angle may affect Pvein VTI net more than the Pvein VTI ratio as absolute values are more dependent on the insonation angle [17].

\section{Pvein VTI versus PA PI}

Fetuses with RAS may have pulmonary venous congestion leading to pulmonary arterial hypertension and pulmonary microvascular changes. Szwast et al. [14] investigated pulmonary vasoreactivity in these patients, hypothesizing that fetuses with less reactive pulmonary vascular beds (i.e., with smaller decrease in PA PI with MHO) were experiencing RAS sequelae and pulmonary venous hypertension. They found that normal fetuses and HLHS fetuses without RAS had a notable decrease in PA PI with MHO, but fetuses with RAS did not. In practice, a cutoff of $<10 \%$ vasoreactivity (decrease in PA PI with MHO) is considered abnormal - their diagnostic utility of PA PI with this cutoff was excellent, with $100 \%$ sensitivity and $94 \%$ specificity [14]. Our PA PI findings were drastically different, with poor sensitivity and specificity at that cutoff value. This discrepancy and the small number of patients in each study make it challenging to compare our respective experiences and highlight the need for collaborative work.

Importantly, there are technical challenges to obtaining PA pulsed wave Doppler and subsequently calculating PA PI. We have found signal quality for PA Doppler to be difficult to optimize, particularly the diastolic component. Machine settings such as "wall filter" or "low velocity reject," which affect the inclusion or exclusion of signal near the baseline, can result in inclusion or exclusion of diastolic blood flow, which greatly affects the mean velocity portion of the PI. In our lab - and many others - Pvein spectral Doppler acquisition is standard for every fetal echocardiogram, while PA spectral Doppler acquisition is not. The increased familiarity with acquisition of Pvein Doppler patterns may result in improved quality over PA Doppler patterns. The challenge in interpreting PA PI (and relative ease of measuring Pvein Doppler parameters) is reflected in our interob-

Maternal Hyperoxygenation Testing in Fetal Hypoplastic Left-Heart Syndrome server variability testing, with Pvein VTI ratio ICCs of $>0.9$ and PA PI ICC at baseline of 0.05 . With MHO, PA PI ICC increased to 0.84 , likely due to the increase in diastolic blood flow.

Another potential benefit of Pvein VTI versus PA PI is the proximal hemodynamic disturbance: as Pvein flow is subject to LA hypertension, it could possibly be affected earlier in gestation than changes in pulmonary vasoreactivity. In this population, pulmonary venous hypertension likely occurs prior to pulmonary arterial hypertension, and there is the literature to support more consistent pathologic change in the Pveins than to the PAs. A small pathologic series comparing pulmonary vascular morphology in HLHS with and without RAS demonstrated abnormal pulmonary venous thickening and fibrosis in $5 / 5$ of the RAS group and 2/5 in the non-RAS group, while $3 / 5$ of both the RAS and non-RAS group showed pulmonary arteriolar thickening [18]. Patients with RAS may show pulmonary venous flow reversal before they have microvascular changes, leading to abnormal pulmonary vasoreactivity.

\section{Postnatal Secondary Outcomes}

Due to practice variation surrounding EAS across institutions, we sought clinical markers that could represent the need for EAS rather than the performance of EAS itself. Postnatal ASD gradient was inversely correlated with the Pvein VTI ratio during MHO (and change from baseline), which may reflect the enhanced ability of MHO testing to predict postnatal physiology. However, a higher ASD gradient may reflect increased pulmonary venous return in a stable neonate and does not necessarily reflect need for EAS. We modeled a composite outcome based on the prior literature. Gellis et al. [7] studied fetuses with known restrictive/intact atrial septa using Pvein Doppler (without MHO). They found that "severe neonatal instability" (defined by the composite clinical outcome in Table 3) was associated with the Pvein VTI ratio $<2.7$ (sensitivity $89 \%$, specificity $97 \%$, AUC 0.93 ), similar to the cutoff for EAS of 3.0 in the Cincinnati experience [16]. In our cohort, a positive composite outcome was associated with a lower Pvein VTI ratio at baseline and with MHO, as well as EAS. We did not find a similar relationship using PA PI. This further supports the use of the Pvein VTI ratio for risk assessment in this setting.

There are some limitations to this work. Our singlecenter, retrospective cohort study may be affected by sample bias that limits generalizability. Our cohort had a significant presence of major comorbidities and genetic syndromes, which likely impacted overall mortality, and our findings may be less significant if children with other 
anomalies are excluded. Additionally, this MHO cohort does not reflect the entirety of the HLHS population seen at our institution. Due to the small number of patients in the study, our conclusions should be viewed merely as hypothesis generating. The optimal cutoff of the MHO Pvein VTI ratio of 6.5 should be interpreted with caution as 2 patients who did not undergo EAS were narrowly above this threshold. Although our center does not perform fetal atrial septal decompression, we refer patients to other centers in appropriate cases. Despite our support of this practice, no patients in our cohort consented to this procedure. As described above, our cohort did not include any fetuses with intact atrial septa, which carries a very poor prognosis [19]. In those cases, MHO would not likely augment the standard fetal echocardiogram. The primary outcome of EAS suffers from being a categorical variable that is potentially subject to confounding. The composite clinical outcome, while associated with the Pvein VTI ratio at baseline and with $\mathrm{MHO}$, may not truly reflect the need for EAS as it may also be present in patients without RAS (e.g., in pulmonary overcirculation). Significant differences in PA PI discriminatory power in the current study compared to the Szwast et al. [14] study are difficult to resolve. A larger, multicenter investigation is needed. Finally, all EAS neonates died. Despite being able to identify fetuses with HLHS who require EAS, clinical outcomes of these patients are still quite poor, likely related to abnormal pulmonary parenchymal architecture and lymphatic channels which develop in utero [20]. The ability to identify these fetuses may not improve the ultimate clinical outcome but may help direct counseling and prompt referral for fetal atrial septoplasty.

\section{Conclusion}

In this study of fetuses with HLHS, the Pvein VTI ratio (at baseline and with $\mathrm{MHO}$ ) reliably identified neonates who underwent EAS. Pvein VTI ratio response to MHO may identify these neonates with higher specificity than PA PI. In our cohort, optimal sensitivity and specificity in identifying EAS neonates were achieved using a tiered approach. Those with a clearly low $(<3)$ Pvein VTI ratio were identified as high-risk for EAS, those with a clearly high ( $\geq 7)$ Pvein VTI ratio were identified as low-risk, and Pvein VTI ratio response to MHO was used to discriminate the intermediate group. Based on these preliminary data, a prospective multicenter study with standardized fetal MHO testing and postnatal management is warranted to assess whether MHO testing is superior to standard fetal echocardiography in identifying fetuses who require EAS and to assess accuracy and reliability of the Pvein VTI ratio and PA PI in this setting.

\section{Acknowledgment}

The authors would like to thank the collaborators and members of the Fetal and Pregnancy Health Program at Lucile Packard Children's Hospital for their support with the study.

\section{Statement of Ethics}

This study was approved by the Stanford University Institutional Review Board prior to study initiation and conducted in accordance with the ethical standards for human research established by the Declaration of Helsinki. The Stanford University IRB protocol no. is 50650. The IRB waived the authors from obtaining informed consent because of the retrospective data collected. Approval was obtained from the Stanford University Institutional Review Board prior to study initiation.

\section{Conflict of Interest Statement}

The authors have no conflicts of interest to declare.

\section{Funding Sources}

No external funding was received to support the research in connection with this article.

\section{Author Contributions}

Christopher Mardy, MD, conceived of the project (with faculty mentor Shiraz A. Maskatia, MD), collected and analyzed the data, and wrote the manuscript. Shiraz A. Maskatia, MD, conceived of the project as mentioned, collected data for the purposes of interobserver variability, and provided major edits to the manuscript. Michelle Kaplinski, MD, and Theresa A. Tacy, MD, provided insight into MHO testing, pulmonary artery, and Pvein Doppler and edited the manuscript. Lynn Peng, MD, helped identify patients who underwent EAS, provided insight into patient selection prior to that procedure, and edited the manuscript. Yair J. Blumenfeld, MD, helped identify patients and edited the manuscript. David M. Kwiatkowski, MD, provided insight into the clinical assessment of neonates with RAS and edited the manuscript.

\section{Data Availability Statement}

The data that support the findings of this study are available from the corresponding author upon request. 


\section{References}

1 Michelfelder E, Gomez C, Border W, Gottliebson W, Franklin C. Predictive value of fetal pulmonary venous flow patterns in identifying the need for atrial septoplasty in the newborn with hypoplastic left ventricle. Circulation. 2005 Nov;112(19):2974-9.

2 Tweddell JS, Hoffman GM, Mussatto KA. Improved survival of patients undergoing palliation of hypoplastic left heart syndrome: lessons learned from 115 consecutive patients. ACC Curr J Rev. 2003 Jan;12(1):95-6.

3 Glatz JA, Tabbutt S, Gaynor JW, Rome JJ, Montenegro L, Spray TL, et al. Hypoplastic left heart syndrome with atrial level restriction in the era of prenatal diagnosis. Ann Thorac Surg. 2007 Nov;84(5):1633-8.

4 Marshall AC, van der Velde ME, Tworetzky W, Gomez CA, Wilkins-Haug L, Benson CB, et al. Creation of an atrial septal defect in utero for fetuses with hypoplastic left heart syndrome and intact or highly restrictive atrial septum. Circulation. 2004 Jul;110(3):253-8.

5 Hamzah M, Othman HF, Baloglu O, Aly H. Outcomes of hypoplastic left heart syndrome: analysis of national inpatient sample database 1998-2004 versus 2005-2014. Eur J Pediatr. 2020 Feb;179(2):309-16.

6 Schneider K, Hahn E, Statile C, Hirsch R, Khoury PR, Cnota J, et al. Pulmonary venous Doppler patterns and midterm outcomes in fetuses with left-sided obstructive lesions and restrictive atrial septum. Prenat Diagn. 2020 Jun;40(7):776-84.

7 Gellis L, Drogosz M, Lu M, Sleeper LA, Cheng $\mathrm{H}$, Allan $\mathrm{C}$, et al. Echocardiographic predictors of neonatal illness severity in fetuses with critical left heart obstruction with intact or restrictive atrial septum. Prenat Diagn. 2018; 38(10):788-94.
8 Vlahos AP, Lock JE, McElhinney DB, van der Velde ME. Hypoplastic left heart syndrome with intact or highly restrictive atrial septum: outcome after neonatal transcatheter atrial septostomy. Circulation. 2004 May;109(19): 2326-30.

9 Lowenthal A, Kipps AK, Brook MM, Meadows J, Azakie A, Moon-Grady AJ. Prenatal diagnosis of atrial restriction in hypoplastic left heart syndrome is associated with decreased 2-year survival. Prenat Diagn. 2012;32(5): $485-90$.

10 Sivarajan V, Penny DJ, Filan P, Brizard C, Shekerdemian LS. Impact of antenatal diagnosis of hypoplastic left heart syndrome on the clinical presentation and surgical outcomes: the Australian experience. J Paediatr Child Health. 2009 Mar;45(3):112-7.

11 Jantzen DW, Moon-Grady AJ, Morris SA, Armstrong AK, Berg C, Dangel J, et al. Hypoplastic left heart syndrome with intact or restrictive atrial septum: a report from the international fetal cardiac intervention registry. Circulation. 2017 Oct;136(14):1346-9.

12 Saul D, Degenhardt K, Iyoob SD, Surrey LF, Johnson AM, Johnson MP, et al. Hypoplastic left heart syndrome and the nutmeg lung pattern in utero: a cause and effect relationship or prognostic indicator? Pediatr Radiol. 2016 Apr;46(4):483-9.

13 Herrmann JL, Irons ML, Mascio CE, Rychik J, Spray TL, Gaynor JW, et al. Congenital pulmonary lymphangiectasia and early mortality after stage 1 reconstruction procedures. Cardiol Young. 2017 Sep;27(7):1356-60.

14 Szwast A, Tian Z, McCann M, Donaghue D, Rychik J. Vasoreactive response to maternal hyperoxygenation $(\mathrm{MH})$ in the fetus with hypoplastic left heart syndrome (HLHS). Circ Cardiovasc Imaging. 2010 Mar;3(2):172-8.
15 Chintala K, Tian Z, Du W, Donaghue D, Rychik J. Fetal pulmonary venous Doppler patterns in hypoplastic left heart syndrome: relationship to atrial septal restriction. Heart. 2008 Nov;94(11):1446-9.

16 Divanović A, Hor K, Cnota J, Hirsch R, Kinsel-Ziter M, Michelfelder E. Prediction and perinatal management of severely restrictive atrial septum in fetuses with critical left heart obstruction: clinical experience using pulmonary venous Doppler analysis. J Thorac Cardiovasc Surg. 2011 Apr;141(4):988-94.

17 Lenz F, Chaoui R. Reference ranges for Doppler-assessed pulmonary venous blood flow velocities and pulsatility indices in normal human fetuses. Prenat Diagn. 2002;22(9): 786-91.

18 Graziano JN, Heidelberger KP, Ensing GJ, Gomez CA, Ludomirsky A. The influence of a restrictive atrial septal defect on pulmonary vascular morphology in patients with hypoplastic left heart syndrome. Pediatr Cardiol. 2002 Mar;23(2):146-51.

19 Rychik J, Rome JJ, Collins MH, DeCampli WM, Spray TL. The hypoplastic left heart syndrome with intact atrial septum: atrial morphology, pulmonary vascular histopathology and outcome. J Am Coll Cardiol. 1999 Aug; 34(2):554-60.

20 Vida VL, Bacha EA, Larrazabal A, Gauvreau K, Thiagaragan R, Fynn-Thompson F, et al. Hypoplastic Left Heart Syndrome With Intact or Highly Restrictive Atrial Septum: surgical Experience From a Single Center. Ann Thorac Surg. 2007 Aug;84(2):581-5; discussion 586 